\title{
Biodegradation of PAHs by Trametes hirsuta zlh237 and Effect of Bioaugmentation on PAHs-Contaminated Soil
}

\author{
Jing Zhen ${ }^{1}$, Jiwen Wang', Lei Li ${ }^{1}$, Zhimin Du ${ }^{1,2 *}$, Guanjie Li ${ }^{1}$, Wenling Yang1 \\ ${ }^{1}$ Key Laboratory of Microbial Engineering at the Institute of Biology, Henan Academy of Sciences, Zhengzhou 450008, \\ People's Republic of China \\ ${ }^{2}$ School of Environmental Engineering, Henan University of Technology, Zhengzhou, 450001, China
}

Received: 20 April 2021

Accepted: 11 October 2021

\begin{abstract}
This study aimed to investigate the biodegradation of three distinct PAHs: Phenanthrene (Phe), Pyrene $(\mathrm{Pyr})$, and Benzo[a]pyrene $(\mathrm{BaP})$ by using fermentation broth from T. hirsuta culture medium. The results revealed that 2,5-xylidine ameliorates laccase production in $T$. hirsuta fermentation medium. After 6 days of incubation, T. hirsuta zlh237 fermentation broth (6-day-FFB) showed a higher degradation rate for three PAHs than 11-day fermentation broth (11-day-FFB). Final Phe, Pyr, and $\mathrm{BaP}$ degradation rates of 6 -day-FFB were $89.35 \%, 85.92 \%$, and $89.45 \%$, respectively, on the $5^{\text {th }}$ day of incubation. The PAHs biodegradation rate of 6-day-FFB in natural soil samples were lower than in sterilized soil. The Phe, Pyr, and BaP degradation rates of 6 -day-FFB were $54.49 \%$, 46.76\%, and $51.93 \%$ in sterilized soil samples, and $39.37 \%, 34.00 \%, 33.99 \%$ in natural soil samples, after 15 days incubation, respectively. The high-throughput sequencing analysis revealed that the 6-dayFFB altered the bacterial community structure and enhanced microbial biodiversity of contaminated soil. The PAHs-contaminated soils incubated with 6-day-FFB showed significant increase in well color development than the controls. Principal Component Analysis of Biolog data differentiated the effect of 6-day-FFB and sterilized 6-day-FFB on contaminated soils. It implied that T. hirsuta zlh237 restored the microbiological functioning of the PAHs contaminated soils.
\end{abstract}

Keywords: biodegradation, microbial functional diversity, Trametes hirsuta, bioaugmentation

\section{Introduction}

Polycyclic Aromatic aromatic hydrocarbons
Hydrocarbons derived from
(PAHs) are natural or

*e-mail: duzhimin324@163.com

anthropogenic sources and widely distributed in terrestrial and aquatic environments [1]. Natural PAH sources include forest and rangeland fires, oil seeps, volcanos, and tree exudates. A remarkable amount of PAHs are released from anthropogenic sources such as petroleum [2]. Apart from being ubiquitous toxic pollutants, PAHs are mutagenic as well as carcinogenic and pose a significant threat to the environment $[3,4]$. 
The PAHs are primarily degraded by the microbes; however, other degradation processes, such as adsorption, volatilization, photolysis, and chemical degradation, also play a substantial role $[5,6]$. Interestingly, ligninolytic fungi, specifically white-rot fungi (WRF), degrade PAHs effectively due to their extracellular enzymes with low substrate specificity [7]. The degradation activity is dependent on the lignindegrading system of these fungi. The ligninolytic system primarily comprises of laccase, lignin peroxidase (LiP), manganese peroxidase (MnP), and hydrogen peroxide generating oxidases. but the oxidation of PAHs by laccases is considered a critical step in this process [8].

Laccases (p-diphenol oxidase, EC 1.10.3.2) belong to a blue multi-copper oxidase group of enzymes widely distributed in higher plants, bacteria, fungi, and insects. Laccase catalyzes monoelectronic oxidation of a broad spectrum of substrates with the concomitant reduction of oxygen to water without the formation of harmful intermediates. Thus, laccases effectively treat or remove industrial and environmental pollutants [9] released due to textile dye transformation, food technological uses, biosensors, waste detoxification, PAHs degradation, and so on $[10,11]$. All these characteristics make laccase an exciting option for the bioremediation of pollutants [12].

The genus Trametes of white-rot fungi is one of the most efficient lignin-degrading genera. Trametes versicolor degrades PAHs effectively. Previous reports have accounted for 12 distinct PAHs' oxidation by this fungus [13]. Trametes hirsuta, notably, is a promising candidate for laccase production [14]. The T. hirsuta culture under the optimal fermentation parameters, after an 3-day incubation period, demonstrated maximum laccase activity of $65.4 \mathrm{U} / \mathrm{mL}$. Trametes hirsuta laccases have been used to decolorize and detoxify textile dyes [15]. Trametes hirsuta D7, a new fungus isolated from Indonesian peat swamp forest, capable of degrading phenanthrene, chrysene, and BaP. The strain showed good performance in the metabolism of phenanthrene [16].

In the current study, we investigated the fermentation broth from Trametes hirsuta zlh237 and PAH's degradation by T. hirsuta zlh237. Microbes in contaminated soil enhance the degradation process; however, the efficiency of this process depends on the composition and catabolic activity of bacterial communities [17]. The soil microbial diversity serves as a useful index for assessing the impact of pollution on soil health [18]. However, the changes in the microbial community structure and diversity that occur when laccase is added to contaminated soil for PAHs remediation remains unexplored. Therefore, in this study, we employed the Biolog plate technique to examine the bioaugmentation effect of $T$. hirsuta zlh237 inoculum on microbial activities in the PAH-contaminated soil. The altered microbial community structure, as a function of treatment type, was assessed using high-throughput sequencing. This study demonstrates the preliminary assessment of white-rot fungus (T. hirsuta zlh237) on PAH degradation.

\section{Materials and Methods}

\section{Fungal Strains for Laccase Production}

T. hirsuta zlh237 (CGMCC No. 11811) was screened from white-rot fungi cultures. T. hirsuta zlh237 culture was confirmed through the ITS internal sequencing analysis coupled with the morphological characterization of fungal cultures. This T. hirsuta zlh237 culture was used further in this study for laccase production and PAH biodegradation.

\section{Liquid Culture Conditions for the Fermentation Broth Preparation}

The T. hirsuta zlh237 fermentation medium was prepared using laccase liquid-state fermentation medium. The fermentation medium contained $\mathrm{NaH}_{2} \mathrm{PO}_{4} \cdot 12 \mathrm{H}_{2} \mathrm{O}(0.39 \mathrm{~g}), \mathrm{MgSO}_{4} \cdot 7 \mathrm{H}_{2} \mathrm{O}(0.5 \mathrm{~g})$, sodium succinate $(1.18 \mathrm{~g}), \mathrm{FeSO}_{4} \cdot 7 \mathrm{H}_{2} \mathrm{O}(0.0315 \mathrm{~g}), \mathrm{CaCl}_{2} \cdot 2 \mathrm{H}_{2} \mathrm{O}$ $(0.1 \mathrm{~g}), \quad \mathrm{MnSO}_{4} \cdot \mathrm{H}_{2} \mathrm{O}(0.035 \mathrm{~g}), \quad \mathrm{CH}_{3} \mathrm{COONa}_{2} \cdot 3 \mathrm{H}_{2} \mathrm{O}$ $(0.408 \mathrm{~g}), \mathrm{CoCl}_{2} \cdot 6 \mathrm{H}_{2} \mathrm{O}(0.06 \mathrm{~g}), \mathrm{ZnSO}_{4} \cdot 7 \mathrm{H}_{2} \mathrm{O}(0.028 \mathrm{~g})$, $\mathrm{CuSO}_{4} \cdot 5 \mathrm{H}_{2} \mathrm{O}(0.168 \mathrm{~g})$, cornflour $(40.0 \mathrm{~g})$, ammonium tartrate $(0.03 \mathrm{~g}), 2,5$-dimethyl aniline $(0.25 \mathrm{~mL})$, vitamin B1 $(10 \mu \mathrm{g})$, vitamin B2 $(5 \mu \mathrm{g})$, vitamin B6 $(5 \mu \mathrm{g})$ in $1 \mathrm{~L}$ of distilled water. Potato dextrose agar (PDA) was used to culture T. hirsuta zlh 237 at $30^{\circ} \mathrm{C}$ for $96 \mathrm{~h}$ in an incubator. $50 \mathrm{~mL}$ fermentation medium in the Erlenmeyer flask $(250 \mathrm{~mL})$ was inoculated with the five $(1 \mathrm{~mm} \times 1 \mathrm{~mm})$ agar plugs from these PDA plates and incubated overnight at $30^{\circ} \mathrm{C}$ with shaking at $180 \mathrm{rpm}$ in the dark. Later, this medium was centrifuged at $15000 \mathrm{rpm}$ at $4^{\circ} \mathrm{C}$ for $5 \mathrm{~min}$, and the supernatant obtained was treated as a crude laccase. 6 days (6-dayFFB) and 11 days (11-day-FFB) fungus fermentation broth was obtained by shake-flask fermentation without centrifugation.

\section{Analysis of Laccase Activity}

Spectrophotometric determination of laccase activity was performed by using 2,2'-Azino-bis (3-ethylbenzthiazoline-6-sulfonic acid) (ABTS) as a substrate and absorbance of the oxidized product was measured at $420 \mathrm{~nm}$. The assay mixture $(2 \mathrm{~mL})$ contained $500 \mu \mathrm{M}$ ABTS $(0.2 \mathrm{~mL}), 100 \mathrm{mM}$ tartaric acid buffer $(1 \mathrm{~mL})(\mathrm{pH})$, water $(0.78 \mathrm{~mL})$, and $20 \mu \mathrm{L}$ laccase enzyme. One unit of enzyme activity equates to enzyme quantity that oxidizes $1 \mu \mathrm{mol}$ ABTS per min at $30^{\circ} \mathrm{C}$ [19]. 


\section{Biodegradation of PAHs Using the Fungal Fermentation Broth}

Biodegradation of the three PAHs (Pyr, Phe, BaP) was performed using 6-day-FFB and 11-day-FFB. The laccase activity in the fermentation broth was $50 \mathrm{U} / \mathrm{mL}$, and the initial concentration of all three PAHs was $150 \mathrm{mg} / \mathrm{L}$. The required quantities of Pyr, $\mathrm{Phe}$, or $\mathrm{BaP}$ was suspended in $1 \mathrm{~mL}$ of $\mathrm{n}$-hexane and added to autoclaved brown glass vials. The n-hexane in these vials was evaporated under a fume hood, and later $3 \mathrm{~mL}$ FFB was added to these brown glass vials and incubated at $30^{\circ} \mathrm{C}$ on a rotary shaker set at $180 \mathrm{rpm}$. During this degradation process, FFB was sampled at regular intervals to quantitate PAH concentration. Biodegradation of the five different concentrations $(12.5,25,50,100$, and $150 \mathrm{mg} / \mathrm{L})$ of each of the three PAHs was evaluated in the 6-day-FFB. All experiments were carried out in triplicates.

\section{Preparation of PAHs-Contaminated Soil}

The soil samples without PAHs (CK) for the experiment were collected from Zhengzhou, Henan province, northern China. The collected soil was air-dried and sieved $(2 \mathrm{~mm})$ to remove stones and plant debris. The physical and chemical properties of the soil were measured. Soil $\mathrm{pH}$ was 8.25. Soil organic matter (OM), alkali-hydrolyzable nitrogen, available potassium (AN) and available phosphorus (AP) contents were $24.1 \mathrm{~g} / \mathrm{kg}, 91.0 \mathrm{mg} / \mathrm{kg}, 57.0 \mathrm{mg} / \mathrm{kg}$ and $30.1 \mathrm{mg} / \mathrm{kg}$, respectively. Soil was added to the brown glass vial and autoclaved at $121^{\circ} \mathrm{C}$ for $20 \mathrm{~min}$. These sterilized soil samples were spiked respectively with stock solutions of Pyr, Phe, and $\mathrm{BaP}$ in hexane to give nominal concentrations of $50 \mathrm{mg} / \mathrm{kg}$ soil. Then, the spiked soils were left under a fume hood for $48 \mathrm{~h}$ to allow hexane to evaporate. Finally, the contaminated soils were equilibrated below $25^{\circ} \mathrm{C}$ for 2 weeks. The natural soil samples were not autoclaved.

\section{Biodegradation of PAH in the Sterilized and Natural Soil Samples by T. hirsuta zlh237}

Each of these sterilized soil samples (50 g each) was inoculated with $25 \mathrm{~mL}$ of 6-day-FFB, aseptically. Control microcosms were inoculated with $25 \mathrm{~mL}$ sterilized fermentation broth. The water holding capacity of the soil was adjusted to $60 \%$ by using sterile water. These samples were incubated at $30^{\circ} \mathrm{C}$ for 15 days in the dark.

The natural soil samples $(50 \mathrm{~g}$ each) were placed in a $15 \mathrm{~cm}$ diameter flowerpot. $25 \mathrm{~mL}$ of 6-day-FFB was inoculated to each of these artificially PAHcontaminated soil samples (PheBA, PyrBA and BaPBA). $25 \mathrm{~mL}$ of sterilized 6-day-FFB was aseptically inoculated to soil samples (PheCK, PyrCK and BaPCK), and it was treated as control. The water holding capacity of the soil was adjusted to $60 \%$ by using sterile water.
These samples were incubated at $30^{\circ} \mathrm{C}$ for 15 days in the dark.

\section{PAH Extraction and Analysis}

The residual Pyr, Phe, BaP in the vials were extracted with $7 \mathrm{~mL}$-hexane. The extracts were mixed first by vortexing for $10 \mathrm{~min}$ and then on a rotary shaker at $180 \mathrm{rpm}$ for $30 \mathrm{~min}$. This solution was allowed to stand for $30 \mathrm{~min}$ at room temperature, and later $1 \mathrm{~mL}$ upper organic phase was aliquoted for further analysis. PAHs in $1 \mathrm{~g}$ of bulk soil samples were extracted using Soxhlet extractor and n-hexane [20].

The concentrations of each PAH in the extracts were determined using HPLC analysis. Around $20 \mu \mathrm{L}$ of PAHs extract was injected into an injection port (HPLC, Agilent technologies, 1200 series), equipped with a U.V. detector set to $254 \mathrm{~nm}$ (column temperature was $30^{\circ} \mathrm{C}$ ). The extract was separated using a ZORBAX SB-C18 column $(0.46 \mathrm{~mm}$ in diameter and $150 \mathrm{~mm}$ in length). The mobile phase was an 80:20 $(\mathrm{v} / \mathrm{v})$ ratio acetonitrile: water mixture and the run was set to a flow rate of $1 \mathrm{~mL} / \mathrm{min}$.

The percentage of PAH degradation (D\%) was calculated using the following formula:

$$
D \%=100 \times\left[\left(M_{\mathrm{i}}-M_{s}\right) \div M_{i}\right]
$$

where $M_{s}$ represents PAH concentration in each treatment, and $M_{i}$ represents initial PAH concentration.

High-Throughput Sequencing . and Biolog Biodiversity Analysis

DNA was extracted from the soil samples $(0.5 \mathrm{~g})$ using PowerSoil DNA Isolation Kit (MoBio Laboratories, Carlsbad, CA) according to manufacturer's instructions. The quality of DNA was checked by $0.8 \%$ agarose gel electrophoresis. The V3-V4 region of the bacteria $16 \mathrm{~S}$ rRNA gene were amplified using 338f/806r primer. These primers contained a set of 8 -nucleotide barcodes sequence unique to each sample. The amplicon mixture was applied to the MiSeq Genome Sequencer (Illumina, San Diego, CA, USA).

The extraction of high-quality sequences was firstly performed with the QIIME package (Quantitative Insights Into Microbial Ecology) (v1.2.1). Raw sequences were selected based on sequence length, quality, primer and tag. The raw sequences were selected and the lowquality sequences were removed. The unique sequence set was classified into operational taxonomic units (OTUs) under the threshold of $97 \%$ identity using UCLUST. Relative abundance (\%) of individual taxa within each community was estimated by comparing the number of sequences assigned to a specific taxon versus the number of total sequences obtained for a sample. Alpha diversity indices (including No. of OTUs, Chaol and Shannon) were calculated on a subset of 24,684 randomly selected sequences from each sample. 
Soil microbial functional diversity and bacterial activity were examined using Biolog EcoPlates (Biolog, Hayward, CA, USA), as described by Liao et al. [21]. These plates test 31 kinds of $\mathrm{C}$ substrates [22]. Briefly, $10 \mathrm{~g}$ of fresh soil was added to $90 \mathrm{~mL}$ of sterilized saline water $(0.9 \% \mathrm{NaCl} \mathrm{w} / \mathrm{v})$ in the $250 \mathrm{~mL}$ Erlenmeyer flask and shaken for $30 \mathrm{~min}$ at $28^{\circ} \mathrm{C}$ and diluted to $10^{-3} .125 \mu \mathrm{L}$ of this diluted solution was inoculated into the Biolog EcoPlates and incubated at $25^{\circ} \mathrm{C}$ for 7 days. Color development was measured as optical density (O.D.) at $590 \mathrm{~nm}$ with a microplate reader at regular $24 \mathrm{~h}$ intervals. Average well color development (AWCD) was used to evaluate microbial activity in each microplate. AWCD was calculated using the following equation: $A W C D=\Sigma O D_{i} / 31$, where $\mathrm{OD}_{i}$ represents the optical density value of each well. Principal Component Analysis (PCA) was performed on Biolog data at $72 \mathrm{~h}$ using the Canoco software.

\section{Data Analysis}

Analysis of variance was performed using the SPSS software version 19.0 (IBM), and PCA was analyzed using the Canoco software version 4.5.

\section{Results}

\section{Laccase Production from T. hirsuta zlh237}

T. hirsuta zlh237 mycelium formed white cottony mass on the PDA plates at $30^{\circ} \mathrm{C}$ after 4-5 days incubation. Mycelia appeared 2-3 days after inoculation and covered the entire plate after 4-5 days. Mycelia appeared dense and white floss shaped. The laccase

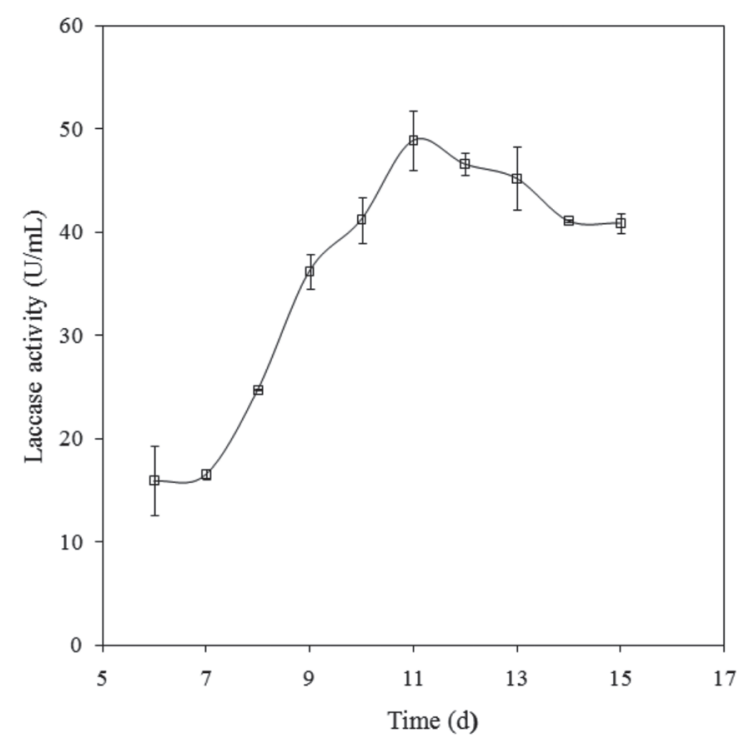

Fig. 1. T.hirsuta zlh237 laccase production profile when cultured in fermentation medium supplemented with 2,5-xylidine as an inducer. Data points represent the average of three replicates. Vertical bars represent standard errors. production from T. hirsuta zlh237 reached its peak on the $11^{\text {th }}$ day (Fig. 1) T. hirsuta $\mathrm{zlh} 237$ produced around $20 \mathrm{U} / \mathrm{mL}$ of laccase when cultured in fermentation medium without inducer, whereas fermentation medium supplemented with 2,5-xylidine as an inducer showed tripled laccase production (Fig. 1), which peaked to $59.8 \mathrm{U} / \mathrm{mL}$ on day 11. An increase in laccase activity intensified the red color of 2,5-xylidine supplemented fermentation medium. It suggests that the 2,5-xylidine supplemented fermentation medium might be correlated to laccase activity.

\section{Biodegradation of PAHs Using T. hirsuta zlh237 Fermentation Broth}

The two different treatments, i.e., 6-dayFFB or 11-day-FFB, degraded Phe, Pyr, and $\mathrm{BaP}$ substantially during five days incubation period (Fig. 2). As shown in Fig. 2a), 6-day-FFB, and 11-day-FFB treatment decreased the Phe concentration to 6.92 and $13.94 \mathrm{mg} / \mathrm{L}$, respectively, on the $5^{\text {th }}$ day. The differences in the degradation rate between these two treatments were significant $(\mathrm{P}<0.05)$. It was significantly higher with 6-day-FFB as compared to 11 -day-FFB on the $5^{\text {th }}$ day. On the first day of incubation, we observed that 6-day-FFB had rapidly degraded Phe and Pyr; however, $\mathrm{BaP}$ was not degraded as effectively as Pyr and Phe (Fig. 2b-c). Around 50\% of Phe and Pyr were degraded after one-day incubation. The maximum 6-day-FFB and 11-day-FFB treatment degradation rate for Phe, Pyr, and $\mathrm{BaP}$ was around $85 \%$ and $80 \%$, respectively, on day 5. The degradation rate of 6-day-FFB treatment for Phe, Pyr, and $\mathrm{BaP}$ was significantly higher than 11-dayFFB. Overall, the results suggest a high degradation efficiency of 6-day-FFB as compared to 11-day-FFB.

Fig. 3 depicted the effects of 6-day-FFB on Phe, $\mathrm{Pyr}$, and BaP biodegradation rate. The initial PAHs concentrations ranged from 12.5-150 $\mathrm{mg} / \mathrm{L}$ with different biodegradation rates. The higher Phe, Pyr, and $\mathrm{BaP}$ concentration resulted in a lower degradation rate. $96.45 \%, 89.42 \%, 84.29 \%, 74.49 \%, 52.33 \%$ of Phe, and $80.35 \%, 80.13 \%, 64.38 \%, 63.20 \%, 62.27 \%$ of $\mathrm{BaP}$ were degraded in the Phe and BaP contaminated samples with initial concentrations of $12.5,25,50$, 100 , and $150 \mathrm{mg} / \mathrm{L}$, respectively (Fig. 3). The different concentrations of Phe and $\mathrm{BaP}$ demonstrated more than $50 \%$ degradation rates. The degradation rate of different Pyr concentrations, i.e., 12.5, 25, 50, 100, and $150 \mathrm{mg} / \mathrm{L}$ were $86.33 \%, 82.33 \%, 83.18 \%, 48.42 \%$, and $35.98 \%$, respectively. The outcomes of our analysis indicate that initial PAH concentration influences the biodegradation rates.

\section{PAHs Biodegradation by T. hirsuta zlh237 in Sterilized and Natural Soil Samples}

T. hirsuta zlh237 increased PAH biodegradation when 6-day-FFB was added to PAH contaminated soil samples from Henan province. This study evaluated 


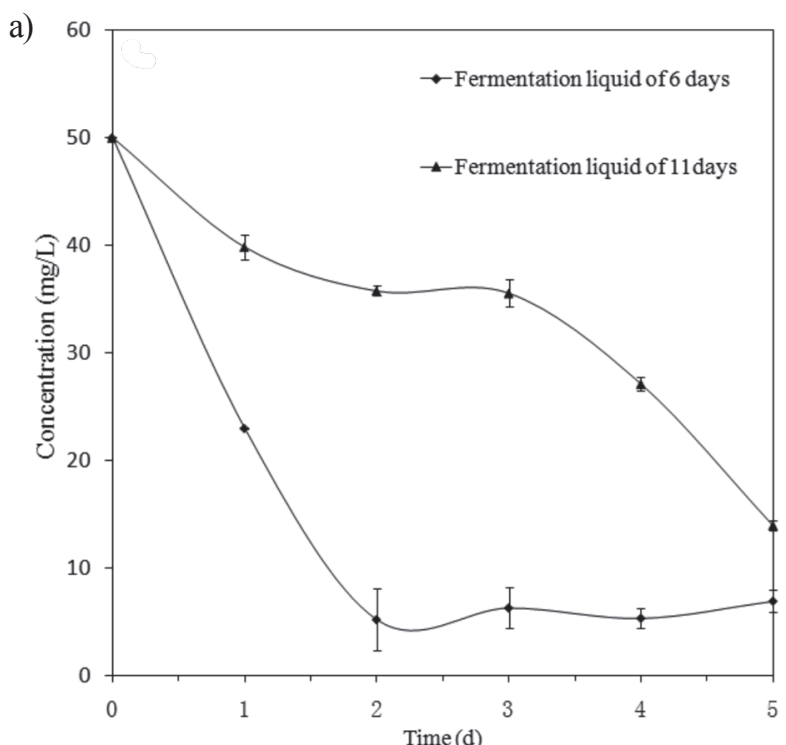

b)
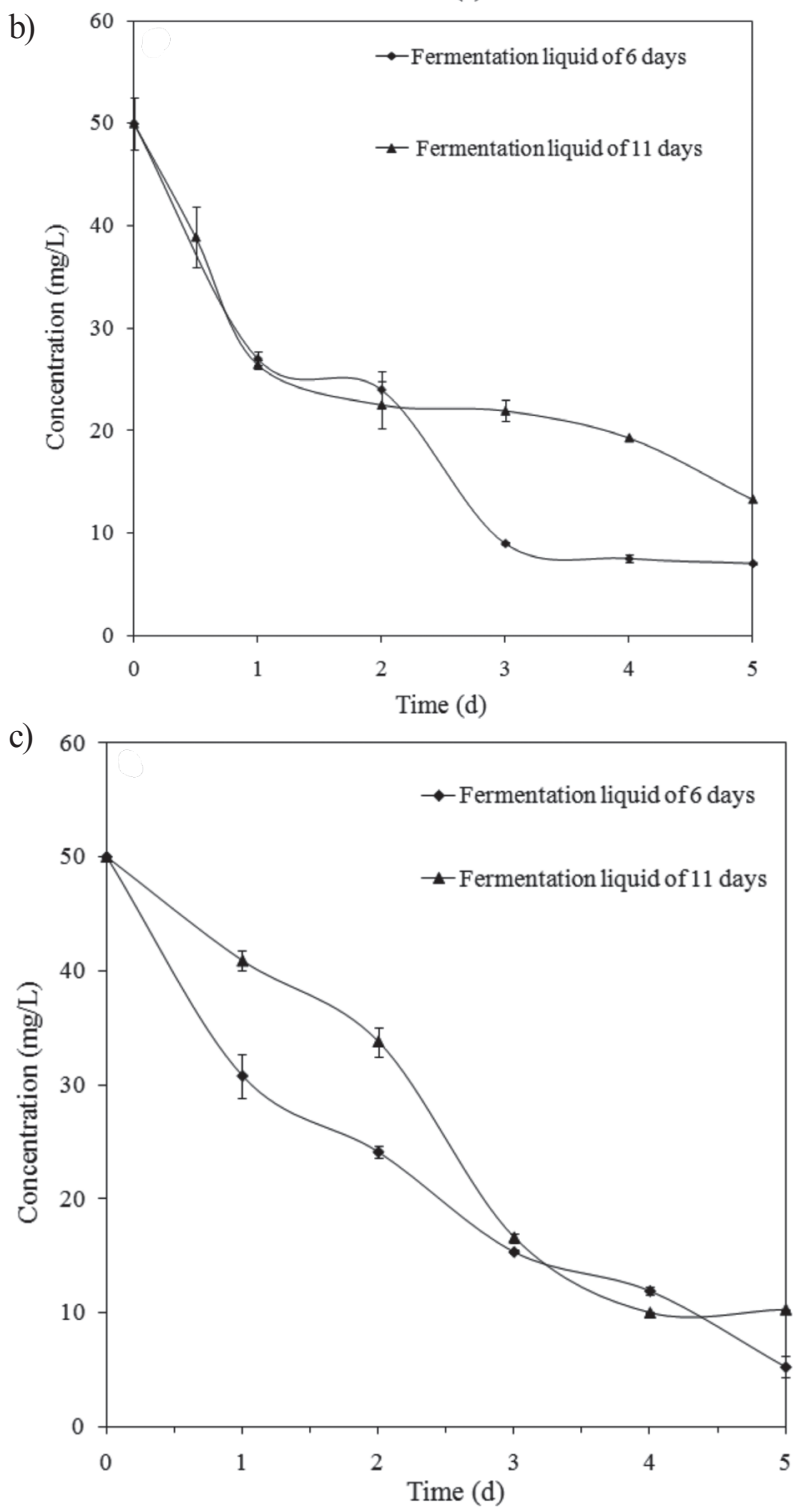

Fig. 2. Biodegradation of the three distinct PAHs (a: Phe, b: Pyr, c: BaP) by T.hirsuta zlh237' 6 days (6-day-FFB) and 11 days (11-day-FFB) fermentation broth. Data points represent the average of three replicates. Vertical bars represent standard errors.

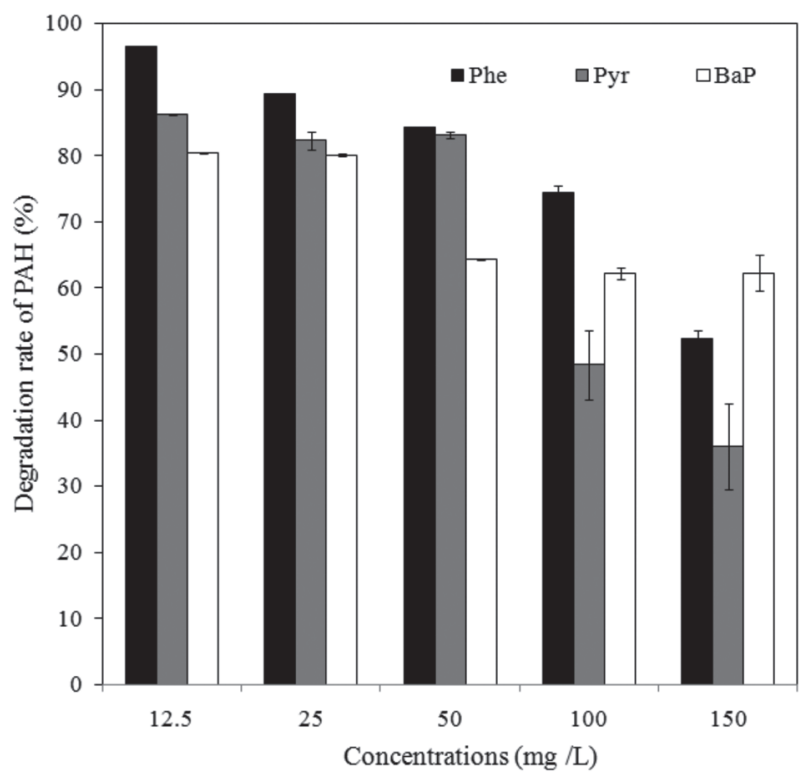

Fig. 3. Phe, Pyr, and $\mathrm{BaP}$ degradation rate after 3 days incubation (a, b, c, d, and e represent 12.5, 25, 50, 100, and $150 \mathrm{mg} / \mathrm{L}$, respectively). Data points represent the average of three replicates. Vertical bars represent standard errors.

the degradation effect of 6-day-FFB on these contaminated soil samples. Fig. 4 demonstrated the biodegradation effect of PAHs on sterilized soil samples. Soil media influenced the degradation effect of PAHs as compared to the direct application of PAHs. We observed that approximately $54.49 \%, 46.76 \%$, and $51.93 \%$ of Phe, Pyr, and $\mathrm{BaP}$ were degraded after 15 days of incubation, respectively (Fig. 4).

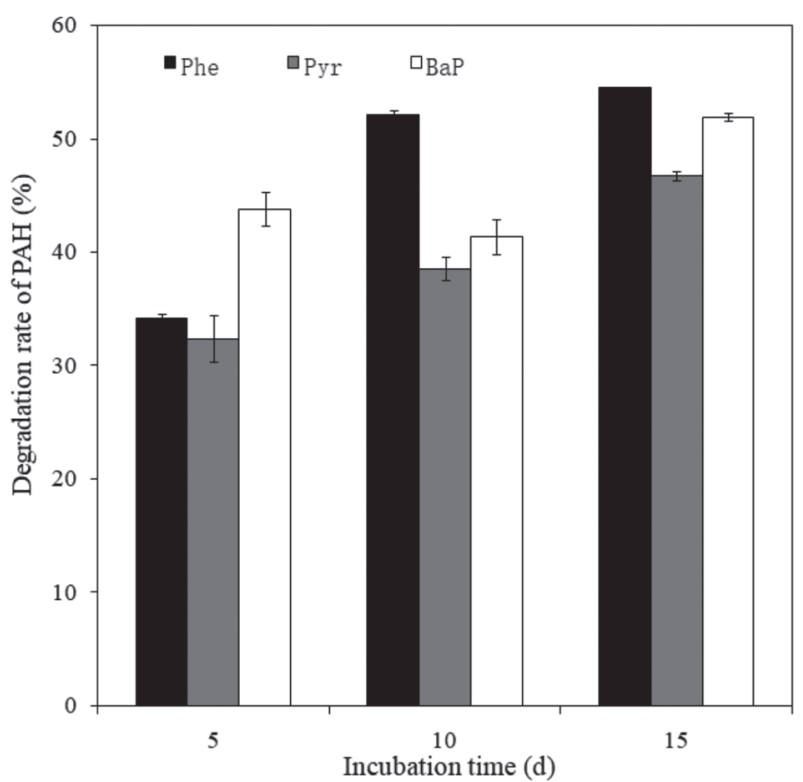

Fig. 4. Phe, Pyr, and BaP degradation rate of 6-day fermentation broth (6-day-FFB) in the sterilized soil after 5-, 10- and 15-days incubation. Data points represent the average of three replicates. Vertical bars represent standard errors. 
Fig. 5 demonstrated the T. hirsuta zlh237 induced PAHs biodegradation effect on the natural soil in the laboratory-scale test. The degradation rate of Phe, Pyr, and $\mathrm{BaP}$ reached $39.37 \%, 34.00 \%$, and $33.99 \%$ after 15-day treatment, respectively, as compared to control (Fig. 5).

\section{Analysis of Microbial Community Composition and Functional Diversity}

The taxonomic distribution at the genus level within different samples was determined, as depicted in Fig. 6. The bacterial diversity at genus level was found to be dramatically different in the PAHs contaminated soil samples incubated with sterilized 6-day-FFB and 6-day-FFB. The bacterial community of CK was mainly composed of Sphingomonas, Skermanella, and Blastococcus, Roseiflexus, Gaiella, Solirubrobacter, Rubrobacter, Microvirga, Streptomyces, Pseudarthrobacter, Asanoa, and Blastococcus. Rubrobacter was the most abundant genus in CK, with an average relative abundance of $7.88 \%$. The relative abundance of Phenylobacterium, $7.44 \%$ and $6.45 \%$, was found to be most in the Phe and Pyr contaminated soil samples, respectively, supplemented with sterilized 6-day-FFB (PheCK, PyrCK). The bacterial community structures changed significantly in the Phe and Pyr contaminated soil supplemented with 6-day-FFB (PheBA, PyrBA). Sphingomonas sp. accounted for $5.25 \%$ and $5.27 \%$ of relative abundances in PheBA and PyrBA, respectively. As per the average relative abundance, the dominant genera of BaP contaminated soil supplemented with 6-day-FFB (BaPBA) were Sphingomonas, RB41, Skermanella, Blastococcus, Gaiella, Solirubrobacter, Rubrobacter, Microvirga, Streptomyces, Pseudarthrobacter, and Nocardioides. The Sphingomonas was the most abundant genus in BaPBA, with an average relative abundance of $8.51 \%$. Besides, the Pseudomonas showed the most relative abundance of $19.17 \%$ in the $\mathrm{BaP}$ soil supplemented with sterilized 6-day-FFB (BaPCK). In comparison, they accounted for $2.85 \%$ of the original soil CK, and $1.19 \%$ in PyrCK. Conversely, it was not detected in PheCK and BaPCK. Phenobacillus was found to be the dominant genus with relative abundances of $7.44 \%$ and $6.45 \%$ in the PheCK and PyrCK, respectively. Pseudomonas was the dominant genus in the BaPCK soil samples with a relative abundance of $19.17 \%$. a)

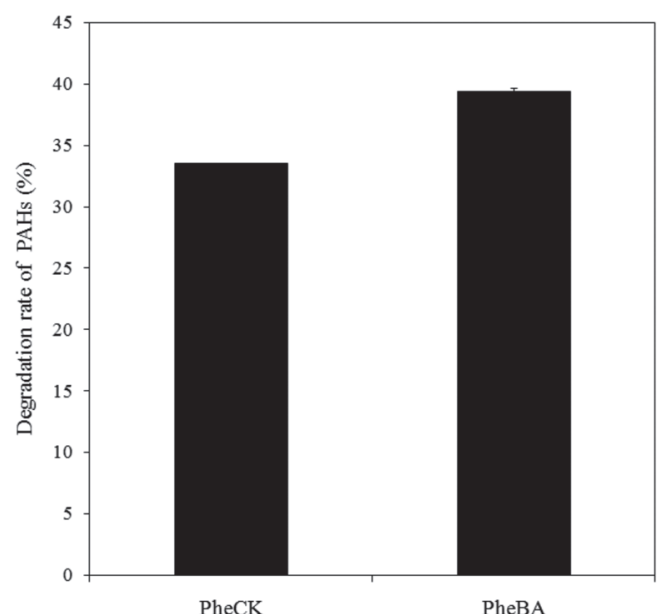

b)

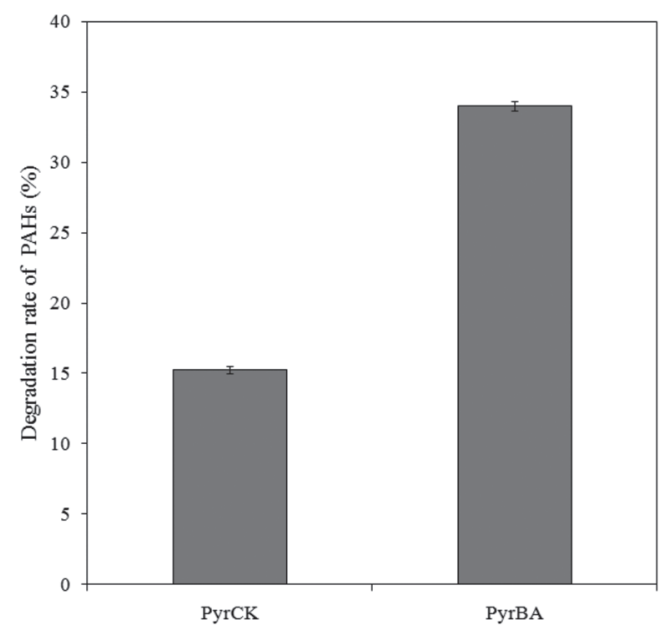

c)

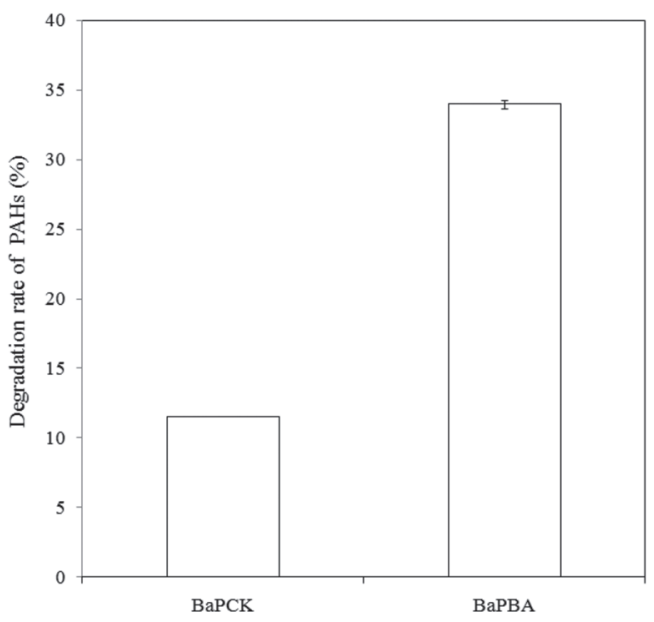

Fig. 5. a) Phe, b) Pyr, and c) BaP degradation rate of 6-day fermentation broth in the natural soil after 15 days incubation. Data points represent the average of three replicates. Vertical bars represent standard errors. 

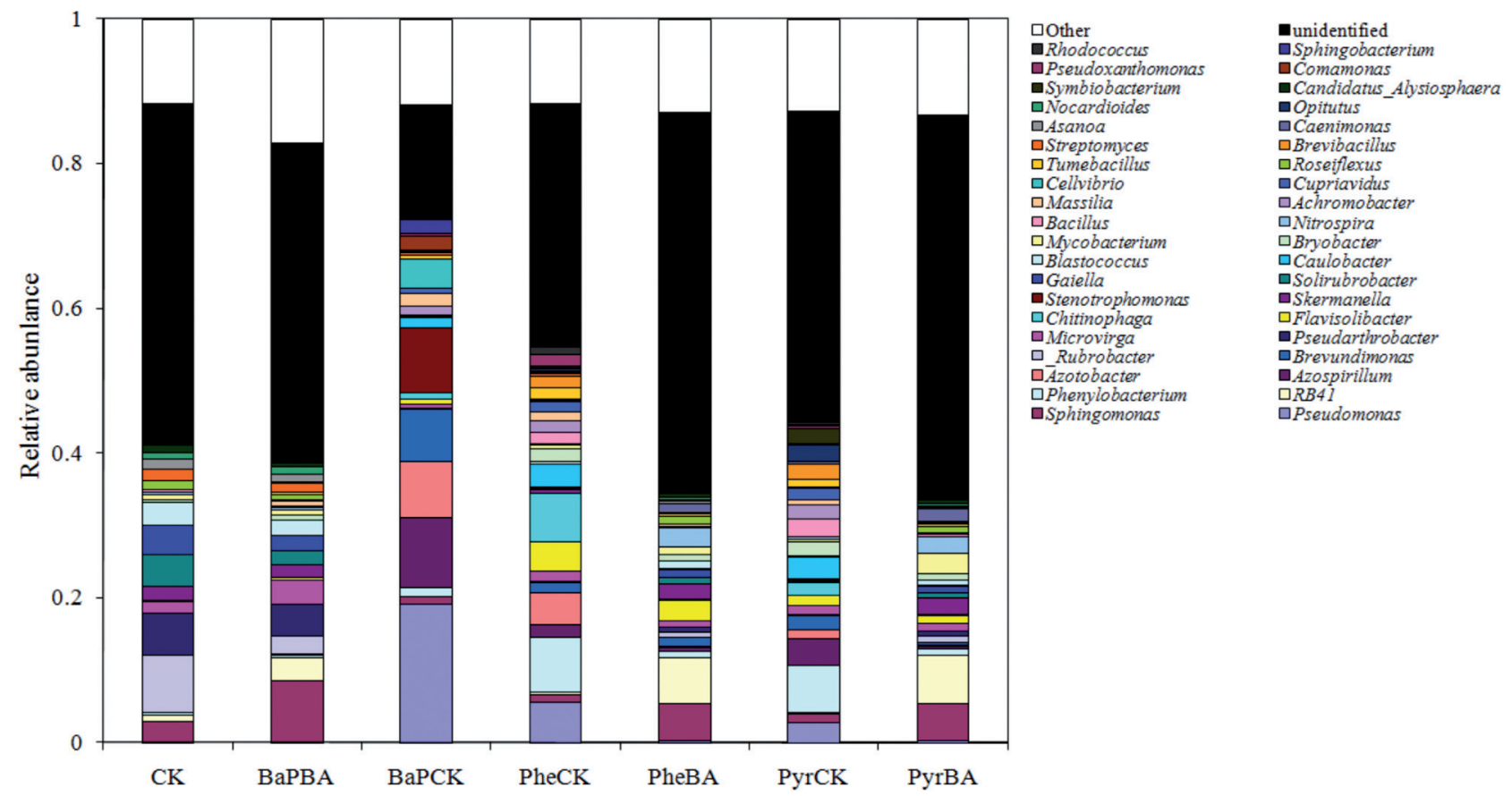

Fig. 6. Bacterial communities and distribution of the microbial genus in the soil samples.

The $\alpha$-diversity indices of bacteria within different samples are shown in Table 1. After removing lowquality reads, 37359, 28609, 29249, 20686, 37996, 12685, and 17974 bacterial sequences were obtained in the CK, PheBA, PheCK, PyrBA, PyrCK, BaPBA, and $\mathrm{BaPCK}$ samples, respectively. The coverage was higher than $96 \%$ for all the samples, indicating that the bacterial sequencing depths were rational and that more sequencing is likely to yield a few additional species.

The sequences were then classified into OTUs at a clustering threshold of $97 \%$ using the CDHIT method. The results indicated that among all the samples, the bacterial OTU numbers were highest in the Phe and Pyr treatments.

The $\alpha$-diversity indices were calculated using the mothur program. The highest values of the biodiversity indices, including Chaol and Shannon, were obtained for the Phe, Pyr, and BaP contaminated soils supplemented with 6-day-FFB. It suggests that the microbial biodiversity of the contaminated soil treated with PheBA, PyrBA, and BaPBA microcosms was better than the other treatments. Thus, T. hirsuta zlh237 fermentation broth addition increased the $\alpha$-diversity of the bacterial communities in PAHcontaminated soil.

Table 1. Effective number and $\alpha$-diversity indices of soil microbial communities in different soil samples.

\begin{tabular}{|c|c|c|c|c|c|}
\hline Soil samples & Final_tags & OTUs & Chao1 & Shannon & Coverage rate \\
\hline CK & 37359 & 1498 & $1729.77 \pm 46.54 \mathrm{~b}$ & $7.97 \pm 0.16 \mathrm{~b}$ & 0.97 \\
\hline PheBA & 28609 & 1178 & $2044.73 \pm 12.55 \mathrm{a}$ & $8.66 \pm 0.23 \mathrm{a}$ & 0.96 \\
\hline PheCK & 29249 & 1765 & $1387.11 \pm 86.90 \mathrm{c}$ & $6.85 \pm 0.23 \mathrm{c}$ & 0.97 \\
\hline PyrBA & 20686 & 970 & $2044.08 \pm 23.69 \mathrm{a}$ & $8.62 \pm 0.09 \mathrm{a}$ & 0.96 \\
\hline PyrCK & 37996 & 1884 & $1307.63 \pm 112.80 \mathrm{c}$ & $6.70 \pm 0.53 \mathrm{c}$ & 0.97 \\
\hline BaPBA & 12685 & 532 & $1941.91 \pm 26.12 \mathrm{a}$ & $8.48 \pm 0.08 \mathrm{a}$ & 0.98 \\
\hline BaPCK & 17974 & 1429 & $824.49 \pm 83.59 \mathrm{~d}$ & $5.42 \pm 0.26 \mathrm{~d}$ & 0.99 \\
\hline
\end{tabular}

Note: CK: initial soil samples; PheCK: Phe-contaminated soil incubated with sterilized 6-day-FFB; PyrCK: Pyr-contaminated soil incubated with sterilized 6-day-FFB; BaPCK: BaP-contaminated soil incubated with sterilized 6-day-FFB; PheBA:

Phe-contaminated soil incubated with 6-day-FFB; PyrBA: Pyr-contaminated soil incubated with 6-day-FFB; BaPBA: BaP-contaminated soil incubated with 6-day-FFB. Different lowercase letters in a column represented significant difference $(\mathrm{P}<0.05)$. 


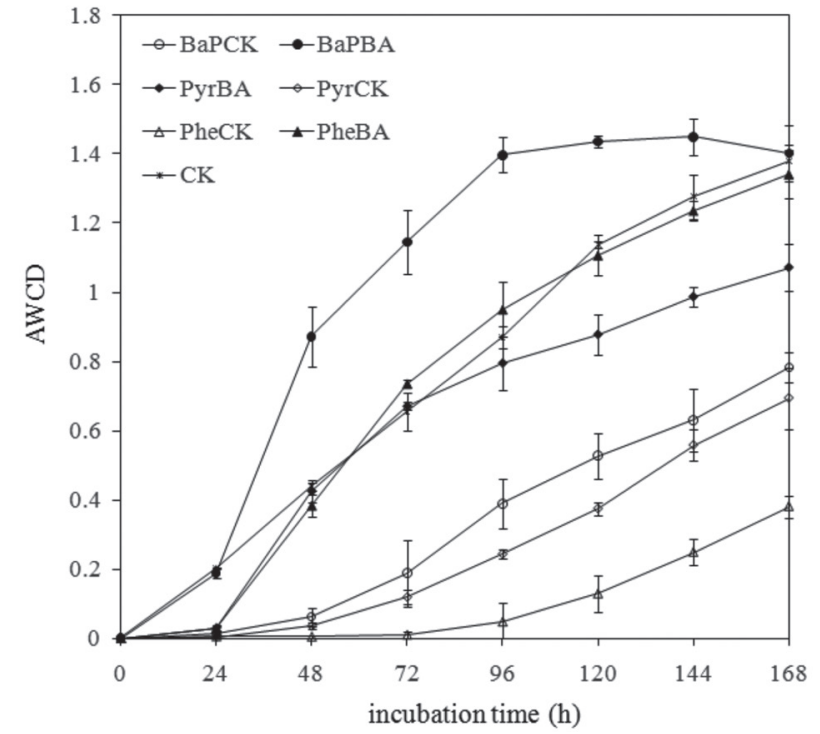

Fig. 7. AWCD for soil samples from the different treatments after 15 days of bioaugmentation. CK represents initial soil samples. Data points represent the average of three replicates. Vertical bars represent standard errors.

AWCD of soil samples with different treatments is represented in Fig. 7. The PAH-contaminated soil samples incubated with 6-day-FFB showed a higher AWCD than PAH-contaminated soil samples incubated with sterilized 6-day-FFB. BaPBA exhibited the highest AWCD. The results implied that the microbial

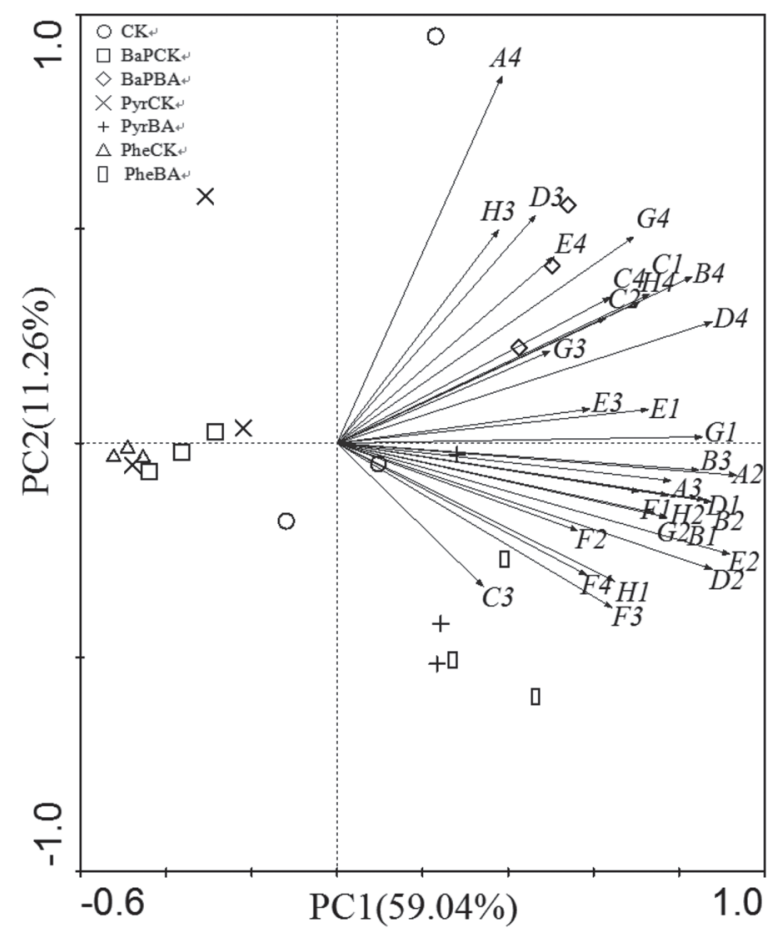

Fig. 8. Principal Components Analysis (PCA) of Biolog data at $72 \mathrm{~h}$ from soil samples after 15 days incubation. CK represents initial soil samples. communities in BaPBA, which was remediated by the T. hirsuta zlh237 strain, were metabolically more active as far as the utilization of carbon substrates is concerned. The AWCD values were BaPBA $>$ PheBA $>$ PyrBA in the treatments of PAHcontaminated soil supplemented with 6-day-FFB. The AWCD of PAH-contaminated soil supplemented with sterilized 6-day-FFB was relatively low.

Principal Component Analysis (PCA) was performed to compare different treatments (Fig. 8). The first and second principal components ( $\mathrm{PC} 1$ and $\mathrm{PC} 2)$ were used to describe the information from 31 carbon sources. Fig. 8 demonstrates the separation of several locations: CK, BaPCK, PheCK, PyrCK, BaPBA, PheBA, PyrBA. $\mathrm{PC} 1$ and $\mathrm{PC} 2$ explained $59.4 \%$ and $11.2 \%$ of the variance in the functional diversity of carbon utilization. The BaPCK, PheCK, and PyrCK soil samples were clustered together, whereas PheBA and PyrBA were clustered together in another sector. The BaPBA samples were independently clustered in the chart, which suggests that the microbial community incubated with 6-day-FFB and sterilized 6-day-FFB were completely different. Soil microbial communities in BaPBA metabolized the substrates, such as 4-Hydroxybenzoic acid (D3), D-Malic acid (H3), L-Threonine (E4), phenylethylamine (G4), L-Phenylalanine (C4), a-Ketobutyric Acid (G3), i-Erythritol (C2), putrescine (H4), tween 40 (C1), L-Asparagine (B4), L-Serine (D4). Soil microbial communities from PheBA and PyrBA mainly utilized 2-Hydroxy Benzoic Acid (C3), itaconic acid (F3), glycyl-L-glutamic acid (F4), $\alpha$-D-Lactose (H1), D-Galacturonic acid (B3), $\beta$-Methyl-D-glucoside as substrates. The samples incubated with 6-day-FFB showed increased utilization level of the substrates as compared to PAH-contaminated soil samples incubated with sterilized 6-day-FFB. PAH-contaminated soil samples supplemented with 6-day-FFB showed more extensive and higher metabolic activity than the soil samples supplemented with sterilized 6-day-FFB.

\section{Discussion}

The adeptness of fungal laccases in oxidizing a wide range of aromatic compounds makes them conspicuous determinants in the environmental bioremediation process [23]. Laccases from different microorganisms have been employed for biodegradation of PAHs, such as a yellow laccase from Leucoagaricus gongylophorus [24], CueO from Escherichia coli [25], and laccase from Trametes villosa [26]. Till now, prior studies had primarily focused on the degradation capacity of laccase determined by the shake flask method. In the current study, we investigated the effect of T. hirsuta zlh237' 6-day-FFB and 11-day-FFB in the PAHs degradation (Phe, Pyr, and $\mathrm{BaP}$ ) in sterilized and natural soil samples. Changes in the microbial community structure in natural soil samples were also assessed. 
The components in fungal culture medium, such as carbon, nitrogen, temperature, $\mathrm{pH}$, and so on, influences laccase production. The addition of various supplements could increase the low yield of laccase. As reported in previous studies, 2, 5-xylidine [27], lignin [28], and veratryl alcohol induces the laccase activity. The outcomes of the current study demonstrated that 2, 5-xylidine supplementation ameliorated T. hirsuta zlh237 growth. Furthermore, an increase in laccase activity intensified the red color of 2,5-xylidine supplemented fermentation medium. It suggests that the 2,5-xylidine supplemented fermentation medium might be correlated to laccase activity. The previous report has accounted for a similar phenomenon in the Pynoporus sanguineus culture medium [27].

In the current study, we found that $T$. hirsuta zlh237 FFB retained its biodegradation capacity until day 5. It might be due to the active T. hirsuta zlh237 in brown bottles containing PAH. The T. hirsuta zlh237 started forming mycelium after 24 hours of incubation. Besides, we found that the degradation rate of PAHs by 6-day-FFB was much higher than that of 11-day-FFB. We speculated that it might be due to the presence of other extracellular enzymes, such as lignin peroxidase and manganese, dependent-peroxidases in 6-day-FFB. Previous studies have stated that these enzymes degrade PAHs efficiently [29]. Natural laccase mediators in the 6-day-FFB might be another reason behind the higher degradation rate of PAHs under lower laccase activity [30].

The outcomes of our analysis indicate that initial $\mathrm{PAH}$ concentration influences the biodegradation rates. The degradation rate of PAHs by 6-day-FFB of T. hirsuta zlh237 decreased as the initial concentration (of PAH) increased, which might be due to the toxicity of aromatic hydrocarbons [31]. In summary, T. hirsuta zlh237 emerged as a promising agent for PAH degradation. Further studies on the T. hirsuta zlh237 mediated degradation of PAH-contaminated soil would be of interest to ensure the efficacy of T. hirsuta zlh237 for soil bioremediation on a large scale.

In this study, we assessed T. hirsuta zlh237 mediated PAHs biodegradation in sterilized and natural soil samples supplemented with 6-day-FFB. The soil media influenced the degradation effect of PAHs. It might be due to the decreased microbial exposure [32] and oxygen concentration caused by the adsorption of PAH on the soil particles, which might have inhibited the laccase activity [33]. Moreover, soil temperature, moisture, redox potential, nutrient availability also hindered the PAH biodegradation [34].

In this study, we observed that the mycelial growth covered the entire soil surface after three days of incubation. It suggests that T. hirsuta zlh237 could remain active in the natural environment and compete with the autochthonous microbial population. The T. hirsuta zlh237 mediated PAH degradation rate in natural soil was lower as compared to the sterilized soil. It might be due to the complex biological structure of the soil that might have mitigated the inoculated microbial population by predation and antagonism from the autochthonous populations [35]. However, biodegradation by introduced fungus demonstrated a higher degradation rate than the natural rate of decontamination and natural attenuation. The degradation rate of Phe, Pyr, and BaP increased to $5.87,18.73,22.44 \%$, respectively, as compared to control soil, after 15-day treatment with 6-day-FFB (Fig. 5). 6-day-FFB showed a better degradation effect on $\mathrm{BaP}$.

The composition of bacterial genera in the community varied with different treatments. In the present study, Sphingomonas and Bacillus been characterized as potentially dominant genera and able to degrade PAHs in contaminated soils [36]. The role of Sphingomonas sp in the biodegradation of aromatic compounds, specifically its efficiency in the low-ring and high-ring PAHs biodegradation, was identified [37]. Sphingomonas sp. accounted for 5.25\%, 5.27\%, and $8.51 \%$ of relative abundances in Phe, Pyr, and BaP contaminated soil samples inoculated with 6-day-FFB, respectively. In comparison, they accounted for $2.85 \%$ of the original soil CK, and $1.19 \%$ in Pyr contaminated soil samples inoculated with sterilized 6-day-FFB. Conversely, it was not detected in Phe and $\mathrm{BaP}$ contaminated soil samples inoculated with sterilized 6-day-FFB. Phenobacillus was found to be the dominant genus with relative abundances of $7.44 \%$ and $6.45 \%$ in the Phe and Pyr contaminated soil samples inoculated with sterilized 6-day-FFB, respectively. Pseudomonas was the dominant genus in the $\mathrm{BaP}$ soil samples with a relative abundance of $19.17 \%$. This study also found that the species and proportion of PAHs degrading bacteria were higher in the contaminated soil inoculated with sterilized 6-day-FFB as compared to 6-day-FFB, but with reduced microbial diversity. It might be due to stress created by high PAHs concentration, which might have hampered low-tolerant microbes due to their inability to adapt to the changing environment, resulting in reduced microbial diversity. Only a few microbial strains that were resistant to PAHs survived and became the dominant bacteria in the soil.

PAHs are toxic environmental pollutants, which negatively influences the microbial activity. Previous studies have investigated the metabolic response of microbial communities using Biolog EcoPlates [38]. The microbial community-level physiological profiles (CLPPs) serves as an apt indicator of the functional dynamics of microbiota in the soil. CLPPs revealed the response of the cultivable portion of the heterotrophic microbial community to carbon substrates. In this study, the physiological profiles of the microbial community indicated differences in functional diversity of soil microbial community when supplemented with sterilized 6-day-FFB and unsterilized 6-day-FFB after 15 days of the remediation process(Fig. 7). These outcomes indicate that the 6-day-FFB supplementation induced higher AWCD in PAH-contaminated soil samples and highest in BaP-contaminated soil samples 
with the highest functional diversity in carbon substrate utilization. In conclusion, bioaugmentation with T. hirsuta zlh237 significantly restored the microbiological diversity of PAHs-contaminated soil.

\section{Conclusions}

6-day-FFB of $T$. hirsuta zlh237, a white-rot fungus, efficiently degraded the Phe, Pyr, and BaP in contaminated soil. The 6-day-FFB Phe, Pyr, and BaP degradation rate was $39.37 \%, 34.00 \%$, and $33.99 \%$ in the natural PAHs-contaminated soil after 15-dayincubation. T. hirsuta zlh237 can increase soil microbiological activity and restore the microbial functioning of the PAHs-contaminated soil. Thus, the outcomes of this investigation suggested that T. hirsuta zlh237 is an excellent candidate with a promising bioremediation strategy for PAHs-contaminated soil.

\section{Acknowledgments}

This research was supported by funds provided by the Basal Research Fund of Henan Academy of Sciences (200605106), Scientific and Technological Project of HeNan province(212102310980, 212102310227), the National Natural Science Foundation of China (32001206) and the Research and Development Project of Henan Academy of Sciences (200405006, 210905004)

\section{Conflict of Interest}

The authors declare no conflict of interest.

\section{References}

1. KAUSHIK C.P., HARITASH A.K. Polycyclic aromatic hydrocarbons (PAHs) and environmental health. Our Earth, 3, 1, 2006.

2. KRISTANTI R.A., HADIBARATA T., AL FARRAJ D.A., ELSHIKH M.S., ALKUFEIDY R.M. Biodegradation mechanism of phenanthrene by Halophilic Hortaea sp. B15. Water, Air, \& Soil Pollution, 229, 1, 2018.

3. LIU S.S., GUO C.L., DANG Z., LIANG X.J. Comparative proteomics reveal the mechanism of Tween 80 enhanced phenanthrene biodegradation by Sphingomonas sp. GY2B. Ecotoxicology and Environmental Safety, 137, 256, 2017.

4. KUMARI S., REGAR R.K., MANICKAM N. Improved polycyclic aromatic hydrocarbon degradation in a crude oil by individual and a consortium of bacteria. Bioresource Technology, 254, 174, 2018.

5. KONG L.L., GAO Y.Y., ZHOU Q.X., ZHAO X.Y., SUN Z.W. Biochar accelerates PAHs biodegradation in petroleum-polluted soil by biostimulation strategy. Journal of Hazardous Materials, 343, 276, 2018.

6. KUPPUSAMY S., THAVAMANI P., VENKATESWARLU K., LEE Y.B., NAIDU R., MEGHARAJ M. Remediation approaches for polycyclic aromatic hydrocarbons (PAHs) contaminated soils: Technological constraints, emerging trends and future directions. Chemosphere, 168, 944, 2017.

7. CAJTHAML T., ERBANOVá P., KOLLMANN A., NOVOTNý Č., ŠAŠEK V., MOUGIN C. Degradation of PAHs by ligninolytic enzymes of Irpex lacteus. Folia Microbiologica, 53, 289, 2008.

8. ZHANG Y., LIN D.F., HAO J., ZHAO Z.H., ZHANG Y.J. The crucial role of bacterial laccases in the bioremediation of petroleum hydrocarbons. World Journal of Microbiology and Biotechnology, 36, 2020.

9. PATEL N., SHAHANE S., SHIVAM, MAJUMDAR R., MISHRA U. Mode of action, properties, production, and application of laccase: a review. Recent Patents on Biotechnology, 13, 1, 2019.

10. THEERACHAT M., TANAPONG P., CHULALAKSANANUKUL W. The culture or co-culture of Candida rugosa and Yarrowia lipolytica strain rM$4 \mathrm{~A}$, or incubation with their crude extracellular lipase and laccase preparations, for the biodegradation of palm oil mill wastewater. International Biodeterioration \& Biodegradation, 121, 11, 2017.

11. VALLECILlOS L., SADEF Y., BORRULL F., POCURULL E., BESTER K. Degradation of synthetic fragrances by laccase-mediated system. Journal of Hazardous Materials, 334, 233, 2017.

12. KADAM A.A., JANG J., LEE D.S. Supermagnetically tuned halloysite nanotubes functionalized with aminosilane for covalent laccase immobilization. ACS Applied Material \& Interfaces, 9, 15492, 2017.

13. ALCALDE M., BULTER T., ARNOLD F.H. Colorimetric assays for biodegradation of polycyclic aromatic hydrocarbons by fungal laccases. Journal of Biomolecular Screening, 7, 547, 2002.

14. SUN K., CHENG X., YU J.L., CHEN L.J., WEI J.J., CHEN W.J., WANG J., LI S.Y., LIU Q.Z., SI Y.B. Isolation of Trametes hirsuta La-7 with high laccase-productivity and its application in metabolism of 17 $\beta$-estradiol. Environmental Pollution, 263, 114381, 2020.

15. ALAM R, ARDIATI F.C., SOLIHAT N.N., ALAM M.B., LEE S.H., YANTO D.H.Y., WATANABE T., KIM S. Biodegradation and metabolic pathway of anthraquinone dyes by Trametes hirsuta D7 immobilized in light expanded clay aggregate and cytotoxicity assessment. Journal of Hazardous Materials, 405, 124176, 2021.

16. HIDAYAT A., YANTO D.H.Y. Biodegradation and metabolic pathway of phenanthrene by a new tropical fungus, Trametes hirsuta D7. Journal of Environmental Chemical Engineering, 6, 2454, 2018.

17. BELL T.H., STEFANI FOP, ABRAM K., CHAMPAGNE J., YERGEAU E., HIJRI M., ST-ARNAUD M. A diverse soil microbiome degrades more crude oil than specialized bacterial assemblages obtained in culture. Applied and Environmental Microbiology, 82, 5530, 2016.

18. CORREA-GARCÍA S., RHEAULT, K., TREMBLAY J., SÉGUIN A., YERGEAU E. Soil characteristics constrain the response of microbial communities and associated hydrocarbon degradation genes during phytoremediation. Applied and Environmental Microbiology, 87, 2020.

19. NIKU-PAAVOLA M.L., KARHUNEN E., SALOLA P., RAUNIO V. Ligninolytic enzymes of the white-rot fungus Phlebia radiata. The Biochemical Journal, 254, 877, 1988.

20. TENG Y., LUO Y.M, SUN M.M, LIU Z.J, LI Z.G, CHRISTIE P. Effect of bioaugmentation by Paracoccus 
sp. strain HPD-2 on the soil microbial community and removal of polycyclic aromatic hydrocarbons from an aged contaminated soil. Bioresource Technology, 101, 3437, 2010.

21. LIAO J., WANG J., JIANG D., WANG M.C., HUANG Y. Long-term oil contamination causes similar changes in microbial communities of two distinct soils. Applied Microbiology and Biotechnology, 99, 10299, 2015.

22. ZAK J.C., WILLIG M.R., MOORHEAD D.L., WILDMAN H.G. Functional diversity of microbial communities: a quantitative approach. Soil Biology and Biochemistry, 26, 1101, 1994.

23. UPADHYAY P., SHRIVASTAVA R., AGRAWAL P.K. Bioprospecting and biotechnological applications of fungal laccase. 3 Biotech, 6, 1, 2016.

24. IKE P.T.L., BIROLLI W.G., DOS SANTOS D.M., PORTO A.L.M., SOUZA D.H.F. Biodegradation of anthracene and different PAHs by a yellow laccase from Leucoagaricus gongylophorus. Environmental Science and Pollution Research, 26, 8675, 2019.

25. ZENG J., ZHU Q.H., WU Y.C., LIN X.G. Oxidation of polycyclic aromatic hydrocarbons using Bacillus subtilis CotA with high laccase activity and copper independence. Chemosphere, 148, 1, 2016.

26. PRASETYO E.N., SEMLITSCH S., NYANHONGO G.S., LEMMOUCHI Y., GUEBITZ G.M. Laccase oxidation and removal of toxicants released during combustion processes. Chemosphere, 144, 652, 2016.

27. VALERIANO V.S., SILVA A.M.F., SANTIAGO M.F., BARA M.T.F., GARCIA T.A. Production of laccase by Pynoporus sanguineus using 2,5 - Xylidine and ethanol. Brazilian Journal of Microbiology, 40, 790, 2009.

28. LAURA M., VICTOR I., MARIA T.L., RAJNI H.-K., GASHAW M. Laccase production by Galerina sp. and its application in dye decolorization. Journal of Yeast and Fungal Research, 5, 13, 2014.

29. KADRI T., ROUISSI T., KAUR BRAR S., CLEDON M., SARMA S., VERMA M. Biodegradation of polycyclic aromatic hydrocarbons (PAHs) by fungal enzymes: A review. Journal of Environmental Sciences, 51, 52, 2017.
30. CAO H.M., WANG C.P., LIU H.B., JIA W.L., SUN H.W. Enzyme activities during Benzo[a]pyrene degradation by the fungus Lasiodiplodia theobromae isolated from a polluted soil. Scientific Reports, 10, 865, 2020.

31. SONG H.G. Biodegradation of aromatic hydrocarbons by several white-rot fungi. Journal of Microbiology, 35, 66, 1997.

32. YUAN S.Y., SHIUNG L.C., CHANG B.V. Biodegradation of polycyclic aromatic hydrocarbons by inoculated microorganisms in soil. Bulletin Environmental Contamination Toxicology, 69, 66, 2002.

33. WAUNG D.W. Optimizing enzymatic preparations of mechanical pulp through the characterization of new laccases and non-productive interactions between enzymes and lignin. Thesis University of Toronto, 2010.

34. KUPPUSAMY S., THAVAMANI P., VENKATESWARLU K., LEE Y.B., NAIDU R., MEGHARAJ M. Remediation approaches for polycyclic aromatic hydrocarbons (PAHs) contaminated soils: Technological constraints, emerging trends and future directions. Chemosphere, 168, 944, 2017.

35. KUPPUSAMY S., THAVAMANI P., MEGHARAJ M., NAIDU R. Bioaugmentation with novel microbial formula vs. natural attenuation of a long-term mixed contaminated soil-treatability studies in solid- and slurry-phase microcosms. Water, Air, \& Soil Pollution. 227, 1, 2015.

36. LI X.N., SONG Y., WANG F., BIAN Y.R., JIANG X. Combined effects of maize straw biochar and oxalic acid on the dissipation of polycyclic aromatic hydrocarbons and microbial community structures in soil: a mechanistic study. Journal of Hazardous Materials, 364, 325, 2019.

37. ASAF S., NUMAN M., KHAN A.L., Al-Harrasi A. Sphingomonas: from diversity and genomics to functional role in environmental remediation and plant growth. Critical Reviews in Biotechnology, 40, 138, 2020.

38. HALEYUR N., SHAHSAVARI E., TAHA M., S.KHUDUR L., KOSHLAF E., OSBORN A.M., S.BALLA A. Assessing the degradation efficacy of native PAH-degrading bacteria from aged, weathered soils in an Australian former gasworks site. Geoderma, 321, 110, 2018. 


\section{Supplementary Material}

Table S1. The specific carbon sources in 6 groups from Biolog-Eco plate experiment.

\begin{tabular}{|c|c|c|c|}
\hline No. & Carbon group & No. & Carbon source \\
\hline \multirow[t]{2}{*}{1} & Amines/amides & G4 & Phenylethylamine \\
\hline & & $\mathrm{H} 4$ & Putrescine \\
\hline \multirow[t]{6}{*}{2} & Amino acids & A4 & L-Arginine \\
\hline & & B4 & L-Asparagine \\
\hline & & $\mathrm{C} 4$ & L-Phenylalanine \\
\hline & & D4 & L-Serine \\
\hline & & E4 & L-Threonine \\
\hline & & $\mathrm{F} 4$ & Glycyl-L-glutamic acid \\
\hline \multirow[t]{10}{*}{3} & Carbohydrates & & Pyruvic acid methyl ester \\
\hline & & H1 & $\alpha$-D-Lactose \\
\hline & & A2 & $\beta$-Methyl-D-glucoside \\
\hline & & B2 & D-Xylose \\
\hline & & $\mathrm{C} 2$ & i-Erythritol \\
\hline & & D2 & D-Mannitol \\
\hline & & E2 & N-Acetyl-D-glucosamine \\
\hline & & G2 & Glucose-1-phosphate \\
\hline & & $\mathrm{H} 2$ & D,L- $\alpha$-Glycerol phosphate \\
\hline & & G1 & D-Cellobiose \\
\hline \multirow[t]{7}{*}{4} & Carboxylic acids & E3 & $\gamma$-Hydroxybutyric acid \\
\hline & & F3 & Itaconic acid \\
\hline & & G3 & $\alpha$-Ketobutyric acid \\
\hline & & $\mathrm{H} 3$ & D-Malic acid \\
\hline & & $\mathrm{F} 2$ & D-Glucosaminic acid \\
\hline & & B3 & D-Galacturonic acid \\
\hline & & $\mathrm{A} 3$ & D-Galactonic acid- $\gamma$-lactone \\
\hline \multirow[t]{2}{*}{5} & Miscellaneous & $\mathrm{C} 3$ & 2-Hydroxybenzoic acid \\
\hline & & D3 & 4-Hydroxybenzoic acid \\
\hline \multirow[t]{4}{*}{6} & Polymers & $\mathrm{C} 1$ & Tween 40 \\
\hline & & D1 & Tween 80 \\
\hline & & E1 & $\alpha$-Cyclodextrin \\
\hline & & $\mathrm{F} 1$ & Glycogen \\
\hline
\end{tabular}

